\title{
JHH Young Investigator Award 2018: Interview with the winner Rachel Climie
}

\author{
Rachel E. Climie ${ }^{1,2}$ \\ Received: 25 April 2019 / Accepted: 25 April 2019 / Published online: 10 May 2019 \\ (c) Springer Nature Limited 2019
}

\section{Could you tell us something about yourself and your unit?}

I am a Research Fellow at Paris Centre de Recherche Cardiovasculaire (PARCC) and a clinical Exercise Physiologist. I completed my $\mathrm{PhD}$ at the Menzies Institute for Medical Research in Tasmania, Australia, under the supervision of Professor James Sharman in 2016. My PhD focused on the clinical and physiological relevance of exercise central haemodynamics in patients with type 2 diabetes (T2D). At PARCC, I am working on the Paris Prospective Study 3, which is an ongoing prospective study in over 10,000 healthy men and women. Within this cohort, I am investigating novel determinants of early vascular ageing and impaired baroreflex sensitivity.

\section{Why did you undertake this research?}

Patients with T2D are at increased risk of cardiovascular disease (CVD) and target organ damage, compared to the general population. The reasons for this are not completely understood, but high blood pressure (BP) and generalized vascular dysfunction are likely to play a role. Furthermore, central BP is more strongly related to target organ damage and CVD risk, compared to brachial BP, and is elevated in patients with T2D. However, specific to patients with T2D, vascular irregularities and CVD risk factors have been shown to have a greater influence on central rather than brachial BP. The reason for

Rachel E. Climie

Rachel.Climie@inserm.fr

1 INSERM U970, Paris Cardiovascular Research Center (PARCC), Paris, France

2 Menzies Institute for Medical Research, University of Tasmania, Hobart, Australia undertaking this systematic review and meta-analysis was to determine whether it is possible to identify the increased risk related to elevated central $\mathrm{BP}$, based on a measure of brachial BP.

\section{What did you learn from this research?}

We learnt that the level of BP amplification from the aorta to the brachial artery is the same in both healthy controls and patients with T2D. However, despite this, patients with T2D have increased central systolic loading due to elevated augmentation pressure and augmentation index, which cannot be identified based on a measure of brachial $\mathrm{BP}$ alone.

\section{Could you expand on the significance of your findings?}

Our findings suggest that patients with T2D are at increased CVD risk due to elevated central systolic stress and that to identify this risk we need to more accurately measure the true BP that people are exposed to. Although cuff BP has been an excellent clinical tool, improvements can be made in terms of measurement precision. This is something that many research groups are currently working on.

\section{Can you tell us about any research your currently undertaking that is related to the paper?}

Patients with T2D display vascular abnormalities compared to their non-diabetic counterparts, such as an increase in stiffness of the large arteries. It is hypothesized that this increase in stiffness is related to an increase in pressure/flow pulsatility that may be transmitted to the microvascular and 
contribute to target organ damage. I am currently investigating how arterial stiffness contributes to elevated pulsatility and in turn, target organ damage in patients with T2D.
Publisher's note: Springer Nature remains neutral with regard to jurisdictional claims in published maps and institutional affiliations. 